\title{
Factores sociodemográficos asociados con los modos de transporte en escolares y adolescentes mexicanos
}

\author{
Luis Ortiz-Hernández*, Ana V. Vega-López y Carolina Ayala-Hilario \\ Departamento de Atención a la Salud, Universidad Autónoma Metropolitana, Unidad Xochimilco, Ciudad de México, México
}

\begin{abstract}
Resumen
Introducción: Los modos activos de transporte (caminar o andar en bicicleta) tienen efectos benéficos para la salud, por lo cual deben identificarse factores que los promuevan. El objetivo de este estudio fue analizar la relación de los modos de transporte utilizados por la población pediátrica mexicana para acudir a la escuela con las características sociodemográficas, socioeconómicas y de inseguridad pública a nivel estatal. Métodos: Se estimó la frecuencia de los modos de transporte utilizados por los escolares y adolescentes para trasladarse a la escuela (con la base de la Encuesta Intercensal 2015). En un análisis ecológico (i.e., entidades federativas como unidades de observación) se obtuvieron correlaciones con estadísticas a nivel estatal con inseguridad alimentaria, nivel de urbanización y mortalidad por muertes violentas. Resultados: El modo de transporte más frecuente fue caminar (66.2\%), seguido por el automóvil (16.2\%) y el transporte público (15.3\%). El transporte activo fue más frecuente en individuos del sexo masculino, escolares, personas de nivel socioeconómico bajo, zonas rurales, la región sur y aquellos cuyo traslado requería menos de 15 minutos. El transporte pasivo fue más frecuente en individuos del sexo femenino, adolescentes, personas de nivel socioeconómico alto y en zonas urbanas. En los estados más urbanizados fue menos frecuente el transporte activo y más prevalente el trasporte motorizado. La inseguridad pública se relacionó negativamente con el uso de bicicleta. Conclusiones: Se requiere mantener o incrementar el uso de modos de transporte activos en la población pediátrica mexicana mediante políticas públicas que mejoren el entorno y garanticen ambientes seguros.
\end{abstract}

Palabras clave: Transporte. Escuela. Niños. Adolescentes. Urbanización. México.

\section{Commuting to school among Mexican schoolchildren and adolescents}

\begin{abstract}
Background: Active commuting (walking or cycling) is associated with benefits to health; thus, it is required to identify factors that promote it. The objective of this study was to analyze the relationship between the transport modes used by Mexican pediatric population to commute to school with sociodemographic and socioeconomic individual characteristics and public insecurity at the state level. Methods: The frequency of transport modes used by schoolchildren and adolescents to commute to school (walking, cycling, private car and public transport) were estimated using the database of the Encuesta Intercensal 2015. In an ecological analysis (i.e., states as observation units) correlations with food insecurity, urbanization level,
\end{abstract}

Disponible en internet: 20-09-2019 Bol Med Hosp Infant Mex.2019;76:225-236 www.bmhim.com 
and violent deaths statistics at state-level were obtained. Results: The most frequent transportation mode was walking (66.2\%), followed by car (16.2\%) and public transportation (15.3\%). Active commuting (walking or cycling) was more frequent in males, schoolchildren, low socioeconomic status, living in rural or southern areas and those who spent $<15$ min to commute. Passive commute was more frequent among females, adolescents, high socioeconomic status, and living in urban areas. In the more urbanized states, active transportation was less frequent, but motorized transportation was more prevalent. Public insecurity was negatively related to cycling. Conclusions: Maintaining or increasing active commuting among Mexican pediatric population is necessary through public policies aimed to improve physical and social environment.

Key words: Transportation. Students. Child. Adolescents. Urbanization. Mexico.

\section{Introducción}

La actividad física es de gran importancia durante la niñez y adolescencia para un óptimo estado de salud ${ }^{1,2}$; de acuerdo con su propósito, se divide en ocupacional, recreativa y para transporte ${ }^{3}$. En la población pediátrica, las formas de transporte activo (caminar o andar en bicicleta) pueden contribuir significativamente a la actividad física total $1^{1,4,5}$. Por ello, es previsible que el tiempo destinado al transporte activo se relacione inversamente con el peso corporal de los niños. En una revisión sobre el tema, se encontró que existe una asociación positiva entre el uso de bicicleta como medio de transporte a la escuela y la aptitud cardiovascular'. Los adolescentes mexicanos que caminaban o usaban bicicleta para ir a la escuela tuvieron menor riesgo de sobrepeso u obesidad (razón de momios $[R M])=0.993 ; p=0.041$ ) respecto de los que no utilizaban estos modos activos de transporte ${ }^{6}$. Asimismo, hay estudios que sugieren que la falta de actividad física se ha convertido en un serio problema de salud pública en países desarrollados, ya que, en los últimos 30 años, la práctica del transporte activo ha disminuido de manera importante ${ }^{2,7}$.

En contraste, el aumento generalizado de la práctica de actividades sedentarias, como la exposición a pantallas (tiempo de exposición a la televisión, computadoras, videojuegos), puede estar relacionado de manera directa con los niveles persistentes de sobrepeso en la población pediátrica ${ }^{8}$. En México, en 2016, hubo una proporción importante de niños y adolescentes con tendencia al sedentarismo, ya que el $77.3 \%$ estuvieron expuestos a pantallas por más de dos horas diarias ${ }^{9}$.

Existe poca información sobre el modo de transporte activo en niños y adolescentes mexicanos. En un estudio realizado a partir de una submuestra de la Encuesta Nacional de Salud y Nutrición $2012^{10}$, se observó que el 70.8\% de adolescentes (10-14 años) utilizaban algún medio de transporte activo. Las variables que se asociaron negativamente con la práctica de dicha actividad fueron el tiempo de traslado, la edad, la escolaridad de la madre, la posesión de un auto en la familia, el nivel socioeconómico, la residencia en zonas urbanas y el norte del país. Sin embargo, en dicho estudio ${ }^{10}$ no se incluyeron a los escolares y tampoco se hicieron estimaciones para cada una de las entidades federativas.

Por lo anterior, se requiere identificar los factores que influyen en el transporte activo para poder diseñar programas de intervención, ya que hay poca evidencia de las causas que influyen en la decisión de utilizar el transporte activo como medio para llegar a la escuela. En países de alto ingreso se ha identificado que los factores como la urbanización, el nivel de riqueza y la inseguridad pública pueden influir en el uso del transporte activo ${ }^{1,8,11}$. En México, es de especial relevancia el posible papel de la inseguridad pública, dado su incremento acelerado en los últimos años. Además, conviene tener información de cada uno de los estados, pues eso permitirá el diseño de políticas y programas regionales o locales.

Por ello, los objetivos de este estudio fueron estimar la frecuencia del uso de los diferentes modos de transporte a nivel nacional y estatal en la población pediátrica mexicana y analizar la relación entre los factores sociodemográficos y de inseguridad pública con los modos de transporte utilizados por escolares y adolescentes. Las características sociodemográficas se analizaron a nivel individual, mientras que para las variables de inseguridad se realizó un análisis ecológico. Con base en la literatura, se esperaba que el uso de transportes activos fuera menor en los estados de mayor inseguridad pública, peores condiciones socioeconómicas y menor ruralidad.

\section{Métodos}

La principal fuente para elaborar el estudio fue la Encuesta Intercensal 2015, cuyo nivel de desagregación permitió hacer dos análisis ${ }^{12}$. El primer análisis se centró en estimar la prevalencia de los diferentes modos de transporte, y la unidad de análisis fueron los 
escolares y adolescentes. El segundo análisis fue de tipo ecológico, con los estados de la república como unidad de análisis; estuvo encaminado a examinar la posible relación de factores sociales y demográficos en la prevalencia de los diferentes modos de transporte. El trabajo fue realizado respetando los acuerdos del artículo 37, párrafo primero de la Ley del Sistema Nacional de Información Estadística y Geográfica en vigor, donde se estipula que los datos utilizados son confidenciales y para uso exclusivamente estadístico.

\section{Estimación de las prevalencias del uso de diferentes transportes}

Se llevó a cabo un estudio de tipo transversal, observacional y analítico a partir de una muestra representativa de población escolar y adolescente de 6 a 15 años de edad en México. Los datos fueron obtenidos a partir de la Encuesta Intercensal 2015, la cual consta de diversos apartados donde se abordan temáticas de características de las viviendas y de la población ${ }^{12}$. Para este análisis se utilizó la base de datos original de la Encuesta Intercensal 2015, en la que estaba contenida la información de 2,746,999 escolares y $1,868,566$ adolescentes.

Las variables seleccionadas para llevar a cabo el análisis fueron el sexo y la edad de los escolares y adolescentes, la asistencia escolar, la escolaridad del jefe de familia, el tamaño de localidad, el tiempo de traslado a la escuela y el medio de traslado empleado para acudir a la escuela. Para fines de esta investigación, solamente se analizaron los datos recabados de niños y adolescentes de 6 a 15 años, por lo que para la variable edad se formaron dos grupos: escolares de 6 a 11 años y adolescentes de 12 a 15 años. Respecto de la asistencia escolar, se consideró el total de la población estimada por el grupo de edad analizado y, a partir de dicha muestra, aquellos que al momento del levantamiento de la encuesta asistían a la escuela.

Para la escolaridad del jefe de familia, se consideró el último grado de estudios aprobado. Los resultados fueron agrupados de la siguiente forma: primaria e inferior, secundaria, bachillerato (incluye preparatoria o bachillerato general, bachillerato tecnológico, estudios técnicos o comerciales con primaria o secundaria o preparatoria terminada y normal con primaria o secundaria terminada), superior (incluye normal de licenciatura, licenciatura, especialidad, maestría o doctorado).

El tamaño de localidad fue agrupado de la siguiente manera: localidades con menos de 2,500 habitantes (rural), de 2,500 a 14,999 habitantes, de 15,000 a
49,999 habitantes, de 50,000 a 99,999 habitantes (urbano) y con más de 100,000 habitantes (ciudades).

En el tiempo de traslado a la escuela, se indagó acerca del tiempo que la persona tardaba regularmente para ir directamente de su vivienda a la escuela, independientemente de la forma o medio empleado. Las opciones de respuesta fueron las siguientes: hasta 15 minutos, de 16 a 30 minutos, de 31 minutos a 1 hora, más de 1 hora.

Con respecto al medio de traslado a la escuela, las respuestas no fueron excluyentes; por consiguiente, era posible circular hasta tres opciones. Las opciones de respuesta fueron caminando, vehículo particular (incluye automóvil, camioneta o motocicleta), transporte público (incluye camión, taxi, combi, colectivo, metro, metrobús y tren ligero), transporte escolar, bicicleta y otros modos.

Se utilizó el módulo de análisis de encuestas del programa Stata versión 13.0 para considerar el diseño complejo de la Encuesta Intercensal (i.e., probabilístico, por conglomerados y estratificado). Con ello, se obtuvieron estimaciones ponderadas por los pesos muestrales asociados con cada observación, y los errores estándar (y los respectivos intervalos de confianza) son estimados por medio del método de linealización de Taylor. Se obtuvieron frecuencias relativas para cada modo de transporte en la población total y de acuerdo con el sexo y edad de los escolares y adolescentes, escolaridad del jefe de familia y tamaño de la localidad. Después, se hicieron las estimaciones para cada estado. Finalmente, se estimó la distribución de acuerdo con el tiempo dedicado al transporte.

\section{Análisis ecológico}

En este análisis, las variables dependientes fueron las prevalencias a nivel estatal de los modos de transporte obtenidas en el análisis previo. Como variables independientes se consideraron diferentes características sociales, demográficas y económicas de los estados, para lo cual se revisaron diversas fuentes. Por lo tanto, en esta base de datos, se contó con 32 unidades de observación (o filas). Las estadísticas descriptivas de estas variables se presentan en la tabla 1.

Para poder analizar la relación de los factores sociodemográficos, las variables independientes fueron inseguridad alimentaria en adultos (tuvo poca variedad en sus alimentos, comió solo una vez o dejó de comer durante todo un día), inseguridad alimentaria en menores (algún menor dejó de desayunar, comer o cenar y algún menor comió menos de lo que debía comer), 
Tabla 1. Estadísticas descriptivas y resultados del análisis de componentes principales de las variables compuestas de la frecuencia de inseguridad alimentaria, ruralidad e inseguridad pública a nivel estatal

\begin{tabular}{|c|c|c|c|}
\hline & Mediana & 01,03 & Pesos* \\
\hline $\begin{array}{l}\text { Inseguridad alimentaria } \\
\text { Algún adulto dejó de desayunar, comer o cenar (\%) } \\
\text { Algún adulto comió menos (\%) } \\
\text { Algún adulto tuvo poca variedad en alimentos (\%) } \\
\text { Algún adulto dejó de comer o lo hizo solo una vez (\%) } \\
\text { Algún adulto sintió hambre pero no comió (\%) } \\
\text { Algún adulto se quedó sin comida (\%) } \\
\text { Algún menor tuvo poca variedad en sus alimentos (\%) } \\
\text { Algún menor comió menos (\%) } \\
\text { A algún menor se le tuvo que servir menos comida (\%) } \\
\text { Algún menor sintió hambre pero no comió }(\%) \\
\text { Algún menor dejó de comer o lo hizo solo una vez (\%) } \\
\text { Algún menor se tuvo que acostar con hambre }(\%)\end{array}$ & $\begin{array}{c}10.2 \\
19.1 \\
29.0 \\
9.4 \\
10.8 \\
10.0 \\
19.0 \\
13.8 \\
13.7 \\
7.1 \\
6.1 \\
6.2\end{array}$ & $\begin{array}{c}9.2,11.9 \\
16.3,22.3 \\
24.9,34.0 \\
8.0,10.6 \\
9.6,12.2 \\
8.9,11.2 \\
15.4,21.4 \\
11.3,15.3 \\
11.8,15.3 \\
6.1,7.7 \\
5.3,6.6 \\
5.4,7.1\end{array}$ & $\begin{array}{l}0.99 \\
0.96 \\
0.86 \\
0.96 \\
0.99 \\
0.98 \\
0.91 \\
0.99 \\
0.99 \\
0.97 \\
0.93 \\
0.96\end{array}$ \\
\hline $\begin{array}{l}\text { Ruralidad } \\
\text { Menos de } 2,500 \text { habitantes }(\%) \\
2,500-14,999 \text { habitantes }(\%) \\
15,000-49,999 \text { habitantes }(\%) \\
50,000-99,999 \text { habitantes }(\%) \\
100,000 \text { y más habitantes }(\%)\end{array}$ & $\begin{array}{c}22.4 \\
16.3 \\
9.2 \\
4.8 \\
42.0\end{array}$ & $\begin{array}{l}12.1,34.1 \\
9.1,22.7 \\
5.5,14.3 \\
2.4,8.1 \\
27.0,62.0\end{array}$ & $\begin{array}{c}0.72 \\
0.90 \\
0.80 \\
0.04 \\
-0.98\end{array}$ \\
\hline $\begin{array}{l}\text { Inseguridad pública } \\
\text { En colonia }(\%) \\
\text { En municipio }(\%) \\
\text { En estado }(\%) \\
\text { En casa }(\%) \\
\text { En escuela }(\%) \\
\text { En carro }(\%) \\
\text { En transporte público }(\%) \\
\text { En calle }(\%)\end{array}$ & $\begin{array}{l}40.4 \\
58.2 \\
65.7 \\
21.0 \\
29.7 \\
40.2 \\
58.0 \\
60.3\end{array}$ & $\begin{array}{l}33.7,45.0 \\
54.5,69.2 \\
56.0,80.1 \\
18.6,24.1 \\
20.8,36.4 \\
36.4,52.0 \\
53.2,70.0 \\
56.1,68.0\end{array}$ & $\begin{array}{l}0.85 \\
0.93 \\
0.88 \\
0.72 \\
0.90 \\
0.92 \\
0.93 \\
0.94\end{array}$ \\
\hline $\begin{array}{l}\text { Problemas comunitarios } \\
\text { Delincuencia cercana a las escuelas (\%) } \\
\text { Pandillerismo (\%) } \\
\text { Robos }(\%) \\
\text { Consumo de alcohol en la calle }(\%) \\
\text { Consumo de drogas en la calle }(\%) \\
\text { Robos/asaltos frecuentes }(\%)\end{array}$ & $\begin{array}{l}24.1 \\
21.1 \\
52.0 \\
64.2 \\
44.0 \\
41.3\end{array}$ & $\begin{array}{l}19.0,28.7 \\
15.2,27.0 \\
40.1,59.0 \\
55.4,68.0 \\
32.2,50.0 \\
29.0,51.1\end{array}$ & $\begin{array}{l}0.94 \\
0.88 \\
0.94 \\
0.52 \\
0.67 \\
0.95\end{array}$ \\
\hline $\begin{array}{l}\text { Muertes } \\
\text { Muertes violentas }(\%) \\
\text { Muertes violentas por accidentes }(\%) \\
\text { Muertes violentas por suicidios }(\%) \\
\text { Muertes violentas por homicidios }(\%)\end{array}$ & $\begin{array}{c}10.1 \\
58.3 \\
9.7 \\
25.0\end{array}$ & $\begin{array}{c}9.4,12.9 \\
49.4,65.2 \\
8.0,12.0 \\
19.0,33.0\end{array}$ & $\begin{array}{c}0.69 \\
-0.84 \\
-0.76 \\
0.98\end{array}$ \\
\hline
\end{tabular}

*pesos en análisis de componentes principales.

01: cuartil 1; 03: cuartil 3.

porcentaje de población en cuatro estratos de urbanización $(<2,500,2,500-14,999,15,000-49,999,50,000$ 99,999 y más de 100,000 habitantes). Esta información fue obtenida de la Encuesta Intercensal ${ }^{12}$.

Las variables independientes asociadas con inseguridad fueron obtenidas a partir de la Encuesta Nacional de Victimización y Percepción sobre Seguridad Pública (ENVIPE 2016) ${ }^{13}$, datos obtenidos por el Instituto Nacional de Estadística y Geografía (INEGI) acerca de causas de mortalidad (2014) $)^{14}$, Indicadores de víctimas visibles e invisibles de homicidio $^{15}$, Panorama epidemiológico y estadístico de la mortalidad en México $(2011)^{16}$ y Tasas de incidencia delictiva por cada 100 mil habitantes $(2016)^{17}$.

Las variables obtenidas de ENVIPE 2016 fueron la percepción de inseguridad (por colonia, municipio o estado) en mayores de 18 años, la percepción de inseguridad en espacios privados (casa, escuela, carro, transporte público y calle), ha dejado de usar transporte público y no permite que los menores salgan solos por temor a ser víctima de delito, delincuencia cercana a la escuela, pandillerismo y robos como problemas comunitarios 
relacionados a violencia, condición de victimización, consumo de alcohol en la calle, consumo de drogas en la calle y robos/asaltos frecuentes como conductas delictivas cercanas a la vivienda (todas las variables anteriores fueron expresadas en porcentajes) ${ }^{13}$.

Las variables obtenidas del informe de mortalidad hasta 2014 fueron el total de muertes violentas y accidentes, suicidios y homicidios como causas de muertes violentas (expresadas en porcentajes) ${ }^{14}$. Del IVVIH-2012 se obtuvo la tasa de muerte por homicidio correspondiente al año $2010^{15}$. Del documento de mortalidad 2011 se obtuvieron las tasas de causas de muertes violentas (accidentes, homicidios y suicidios) $^{16}$.

Dado que las variables que miden el mismo fenómeno y son evaluadas en un mismo estudio (percepción de inseguridad pública en el estado, municipio, colonia, automóvil, de la ENVIPE 2016) pueden estar correlacionadas, se recurrió al análisis factorial con el objetivo de reducir variables y lograr parsimonia en los modelos de regresión. Los resultados de este análisis se presentan en la última columna de la tabla 1, los cuales sirvieron para el cálculo de las siguientes variables compuestas: - Inseguridad alimentaria: incluyó las variables de inseguridad alimentaria en menores y adultos.

- Ruralidad: incluyó los cinco estratos de localidad (expresados en porcentajes).

- Inseguridad pública: incluyó la percepción de inseguridad (por colonia, municipio o estado) y la percepción de inseguridad en espacios privados (casa, escuela, carro, transporte público y calle).

- Cambios en conducta: incluyó las respuestas «ha dejado de usar transporte público, no permite que los menores salgan solos por temor a ser víctima de delito, considera la posibilidad de ser víctima de al menos un delito, posibilidad de ser víctima de robo en transporte público/calle y condición de victimización".

- Problemas comunitarios: incluyó la delincuencia cercana a las escuelas, pandillerismo, robos, consumo de alcohol en la calle, consumo de drogas en la calle y robos/asaltos frecuentes.

- Criminalidad: incluyó la tasa de prevalencia de robo/ asalto en la calle/transporte público, la tasa de prevalencia total de ocurrencia delictiva y la tasa de extorsión.

- Muertes violentas (porcentaje): incluyó el total de muertes violentas y accidentes, suicidios y homicidios como causas de muertes violentas.

- Muertes violentas (tasa): incluyó la tasa de accidentes y las tasas de homicidios como causas de muerte violenta.
Una de las estadísticas que se obtienen en el análisis factorial son los pesos factoriales, los cuales son una estadística de resumen de la contribución de cada variable observada a una variable latente. Por ejemplo, en la última columna de la tabla 1 se puede observar que el peso factorial para la variable de 100,000 habitantes o más fue de -0.98 , mientras que para la variable de 2,500 a 14,999 habitantes fue de 0.90. Los pesos son multiplicados por los valores de cada estado en cada una de las cinco variables observadas, incluidas en la variable latente denominada ruralidad, y los productos son sumados. Por lo anterior, las variables latentes no tienen unidades de medición.

Para conocer la relación entre los medios de transporte utilizados para ir a la escuela y las variables relacionadas con ruralidad, inseguridad alimentaria e inseguridad pública, se utilizaron algoritmos automatizados de eliminación progresiva e introducción progresiva. En el procedimiento de eliminación progresiva se introducen todas las variables en la ecuación, y después se van excluyendo una tras otra conforme tienen la menor correlación parcial con la variable dependiente ( $p>0.100$ como criterio de eliminación). En el procedimiento de introducción progresiva, las variables se incorporan sucesivamente, comenzando por la que tenga la mayor correlación con la variable dependiente; una variable se introduce si predice la variable dependiente $(p<0.050)$.

\section{Resultados}

En la tabla 2, se observa la distribución de la población de acuerdo con las características sociodemográficas. A nivel nacional, el $94.8 \%$ de los escolares y adolescentes mexicanos asistían a la escuela, con una mayor proporción de asistencia del sexo femenino $(95 \%)$, escolares $(97.7 \%)$, aquellos cuyos padres tienen un nivel de estudios superior (98.6\%) y que vivían en zonas urbanas $(96 \%)$.

En relación con el medio de transporte utilizado para acudir a la escuela, a nivel nacional existe una mayor tendencia a utilizar modos de transporte activo: caminando $(66.1 \%)$ y bicicleta (1.6\%), lo cual es importante mantener, e incluso favorecer su incremento. La suma de los modos de transporte pasivo (carro, transporte público y escolar) no superó el $34 \%$.

Quienes más caminaron a la escuela fueron escolares de sexo masculino cuyos padres estudiaron hasta la secundaria y quienes vivían en localidades rurales. El carro fue más utilizado por escolares de sexo femenino cuyos padres tenían estudios de nivel 
Tabla 2. Modo de transporte utilizado para ir a la escuela de acuerdo a características sociodemográficas

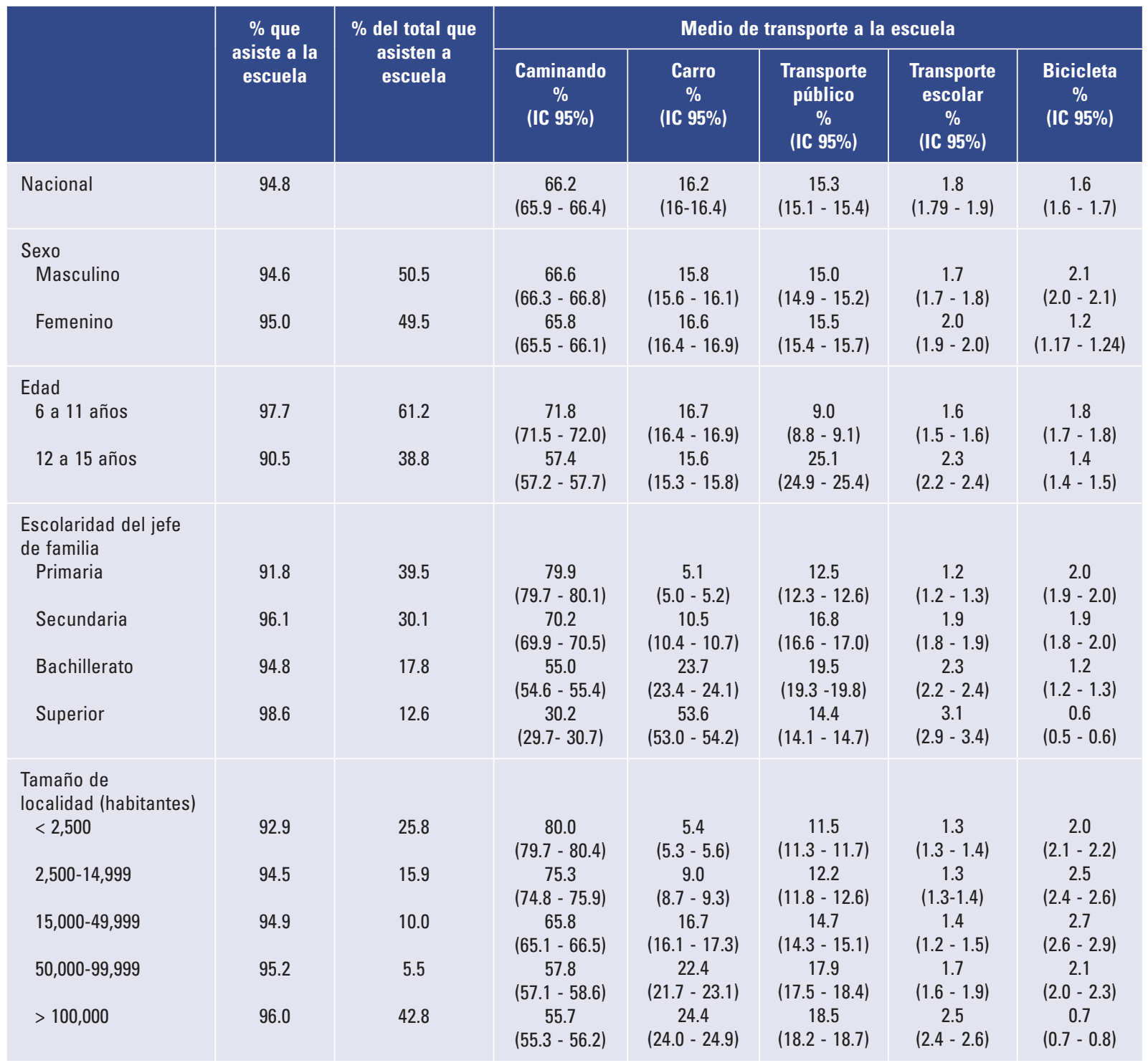

IC: intervalo de confianza.

Fuente: estimaciones propias a partir de la base de datos de la Encuesta Intercensal 2015

superior y quienes vivían en poblaciones con más de 50,000 habitantes. Las adolescentes cuyos padres estudiaron hasta bachillerato y que vivían en localidades con más de 50,000 habitantes usaban con mayor frecuencia el transporte público. El transporte escolar fue más utilizado por adolescentes de sexo femenino con padres con estudios de nivel superior, y que vivían en lugares con más de 100,000 habitantes. El uso de bicicleta fue preferido por escolares de sexo masculino de padres con estudios de nivel básico y que vivían en zonas de entre 2,500 y 49,999 habitantes.
Se analizaron las diferencias por estado en los modos de transporte (Tabla 3). En el caso de los escolares, los estados donde más caminaban fueron Chiapas, Guerrero y Oaxaca, mientras que donde menos lo hacían fueron Baja California Sur, Tamaulipas y Sonora. El uso de bicicleta fue más común en Yucatán, Campeche y Tabasco, contrario a Nuevo León, Querétaro y Chihuahua. Los estados donde más utilizaban carro fueron Baja California Sur, Sonora y Baja California, mientras que en Chiapas, Guerrero y Oaxaca sucedió lo contrario. En la Ciudad de México, Tabasco y Morelos fue donde más utilizaban transporte público, 
opuesto a Aguascalientes, Nuevo León y Zacatecas. Se observó una mayor tendencia al uso de transporte escolar en Nuevo León, Coahuila y Tamaulipas, mientras que, en Guerrero, Quintana Roo y Nayarit, la frecuencia fue menor.

En el caso de los adolescentes (Tabla 4) los estados donde más caminaban fueron Chiapas, Oaxaca y Guerrero, mientras que en Tamaulipas, Coahuila y Baja California Sur fue donde menos lo hacían. El uso de bicicleta fue más común en Yucatán, Campeche y Guanajuato, contrario a Baja California Sur, Nuevo León y Querétaro, con frecuencias menores. Los estados donde más utilizaban carro fueron Baja California Sur, Baja California y Sonora, mientras que, en Oaxaca, Guerrero y Chiapas fue menos común. En la Ciudad de México, Tabasco y Tlaxcala fue donde más utilizaban transporte público, opuesto a Zacatecas, Nuevo León y Aguascalientes. Se observó una mayor tendencia al uso de transporte escolar en Tamaulipas, Nuevo León y Coahuila, mientras que en Guerrero, Quintana Roo y Oaxaca, la frecuencia fue menor.

Del total de personas que se transportaban principalmente caminando, en auto, en bicicleta $u$ otro tipo de transporte, la mayoría empleaba menos de 15 minutos en llegar a la escuela (Tabla 5); quienes usaban el transporte público o escolar, empleaban entre 16 y 30 minutos. La mayoría de los escolares que caminaban, usaban carro, transporte escolar, bicicleta u otro medio empleaban menos de 15 minutos para llegar a la escuela; los que usaban el transporte público, empleaban entre 16 y 30 minutos. De los adolescentes que principalmente caminaban, usaban automóvil, bicicleta u otro tipo de transporte, tardaban hasta 15 minutos en llegar a la escuela; los que se desplazaban en transporte público o escolar tardaban entre 16 y 30 minutos.

Luego de estimar los modelos de regresión lineal con los comandos «hacia adelante» $y$ «hacia atrás» (forward y backward), se observó que aquellos estimados con «hacia atrás» mostraban mejores valores de significación estadística.

Los factores asociados con la elección en el uso de medios de transporte para llevar a la escuela a escolares y adolescentes mexicanos se presentan en la tabla 6. En el análisis bivariado, la prevalencia de caminar a la escuela se asoció positivamente con la inseguridad alimentaria y la ruralidad; mientras que, en el modelo de regresión, solo la última relación se mantuvo. En los adolescentes, tanto en el análisis bivariado como en el modelo de regresión, la prevalencia de caminar se relacionó positivamente con la ruralidad. En ambos grupos de edad, en el análisis bivariado, la prevalencia de uso de automóvil se relacionó negativamente con la inseguridad alimentaria y la ruralidad. Sin embargo, en el modelo de regresión entre los escolares, la prevalencia de uso de auto se relacionó negativamente con la ruralidad y la inseguridad pública, mientras que, en los adolescentes, la relación fue negativa con la inseguridad alimentaria. En ambos grupos, tanto en el análisis bivariado como en el modelo de regresión, la prevalencia de uso de transporte público se relacionó positivamente con la inseguridad pública. En los dos grupos de edad, la prevalencia de uso de transporte escolar se relacionó negativamente con la inseguridad alimentaria. Tanto en escolares como en adolescentes, en el análisis bivariado y en el modelo de regresión, el aumento de las muertes violentas se relacionó con la menor prevalencia del uso de bicicleta.

\section{Discusión}

Un hallazgo positivo del estudio fue que la mayoría de los niños y adolescentes mexicanos caminaban a la escuela, el cual es un modo de transporte activo. Esta forma de transporte debe promoverse para que, por lo menos, la frecuencia con que se utiliza no disminuya. En cambio, el uso de bicicleta fue muy reducido, por lo que debe ser promovido. En contraste, alrededor del 33.3\% de adolescentes y escolares utilizaban algún modo de transporte pasivo (carro, transporte público o escolar). Debe resaltarse que es frecuente el uso de transporte pasivo para recorridos cortos. Por ejemplo, casi dos terceras partes de los usuarios de automóvil privado $(65.8 \%$ en ambos grupos de edad) realizaban trayectos de menos de 15 minutos. En estos casos, convendría alentar la sustitución de medios motorizados por alguna forma de transporte activo.

Respecto a las diferencias por edad, mientras que en los escolares el caminar y el uso de bicicleta fue más alto, en los adolescentes fue más frecuente el uso de transportes público y escolar. Esta diferencia podría ser difícil de reducir si se debe a que los adolescentes se desplazan mayores distancias para los centros escolares. Se tiene que explorar en el futuro a qué factores modificables puede ser atribuida la reducción de los modos de transporte activos que ocurre con la edad. En niños californianos de 5 a 7 años se observó que, conforme aumentaba la edad, era mayor la preferencia por el uso de transporte activo ${ }^{18}$. Sin embargo, en este estudio, se observó lo contrario, ya que los escolares tuvieron menor frecuencia de uso de transporte activo. 
Bol Med Hosp Infant Mex. 2019;76

Tabla 3. Modos de transporte utilizados por escolares para ir a la escuela por entidad federativa

\begin{tabular}{|c|c|c|c|c|c|c|c|}
\hline & \multirow[t]{2}{*}{ Total de niños } & \multirow{2}{*}{$\begin{array}{l}\% \text { que asiste } \\
\text { a la escuela }\end{array}$} & \multicolumn{5}{|c|}{ Medio de transporte a la escuela } \\
\hline & & & $\begin{array}{c}\text { Caminando } \\
(\%)\end{array}$ & $\begin{array}{c}\text { Carro } \\
(\%)\end{array}$ & $\begin{array}{c}\text { Transporte público } \\
(\%)\end{array}$ & $\begin{array}{c}\text { Transporte escolar } \\
(\%)\end{array}$ & $\begin{array}{c}\text { Bicicleta } \\
(\%)\end{array}$ \\
\hline Aguascalientes & 152,787 & 98.5 & 72.8 & 22.5 & 3.1 & 0.6 & 2.1 \\
\hline Baja California & 359,510 & 97.8 & 55.6 & 38.9 & 5.9 & 1.2 & 0.8 \\
\hline Baja California Sur & 76,296 & 97.8 & 47.7 & 45.0 & 5.9 & 2.1 & 0.5 \\
\hline Campeche & 95,949 & 97.2 & 63.5 & 18.8 & 11.5 & 0.2 & 4.5 \\
\hline Coahuila & 325,325 & 98.2 & 58.0 & 29.2 & 7.4 & 5.5 & 0.9 \\
\hline Colima & 74,725 & 97.3 & 66.0 & 26.0 & 7.4 & 0.2 & 1.4 \\
\hline Chiapas & 691,093 & 95.7 & 86.9 & 4.8 & 5.9 & 0.4 & 1.3 \\
\hline Chihuahua & 410,483 & 95.5 & 59.5 & 33.3 & 4.1 & 2.9 & 0.5 \\
\hline Ciudad de México & 740,486 & 98.1 & 65.0 & 16.0 & 18.2 & 2.6 & 0.7 \\
\hline Durango & 203,491 & 98.1 & 73.6 & 18.3 & 6.8 & 1.3 & 1.2 \\
\hline Guanajuato & 675,540 & 98.0 & 69.6 & 16.0 & 10.3 & 1.1 & 4.1 \\
\hline Guerrero & 436,497 & 97.4 & 85.5 & 5.0 & 8.7 & 0.1 & 0.7 \\
\hline Hidalgo & 332,241 & 98.7 & 76.4 & 11.5 & 11.8 & 0.3 & 1.6 \\
\hline Jalisco & 877,175 & 97.5 & 71.9 & 18.8 & 6.9 & 1.4 & 2.0 \\
\hline México & $1,765,672$ & 98.3 & 78.5 & 9.1 & 9.7 & 1.9 & 1.6 \\
\hline Michoacán & 526,331 & 97.3 & 76.7 & 13.8 & 8.2 & 0.4 & 1.7 \\
\hline Morelos & 199,568 & 97.6 & 69.8 & 14.8 & 15.2 & 0.4 & 1.0 \\
\hline Nayarit & 132,701 & 97.8 & 74.0 & 15.3 & 8.3 & 0.1 & 3.6 \\
\hline Nuevo León & 530,722 & 98.7 & 62.6 & 27.3 & 3.4 & 7.1 & 0.3 \\
\hline Оахаса & 479,388 & 96.3 & 85.1 & 5.2 & 6.9 & 0.1 & 1.4 \\
\hline Puebla & 729,289 & 97.6 & 79.3 & 7.7 & 11.1 & 0.8 & 2.0 \\
\hline Querétaro & 227,081 & 98.5 & 68.5 & 19.8 & 10.3 & 2.1 & 0.4 \\
\hline Quintana Roo & 165,372 & 97.4 & 60.9 & 23.2 & 12.4 & 0.1 & 4.2 \\
\hline San Luis Potosí & 315,433 & 98.5 & 74.3 & 17.4 & 5.8 & 0.6 & 3.2 \\
\hline Sinaloa & 321,663 & 98.2 & 64.5 & 26.9 & 5.8 & 1.5 & 2.3 \\
\hline Sonora & 312,802 & 97.2 & 54.0 & 39.9 & 5.0 & 1.3 & 1.2 \\
\hline Tabasco & 271,837 & 97.9 & 62.2 & 15.0 & 17.4 & 0.6 & 4.3 \\
\hline Tamaulipas & 382,775 & 97.6 & 52.5 & 32.5 & 9.3 & 5.4 & 0.9 \\
\hline Tlaxcala & 149,527 & 98.6 & 78.8 & 8.0 & 12.0 & 1.0 & 1.7 \\
\hline Veracruz & 858,846 & 97.5 & 79.5 & 9.7 & 10.2 & 0.1 & 1.0 \\
\hline Yucatán & 219,398 & 98.7 & 56.0 & 22.8 & 9.7 & 0.3 & 8.8 \\
\hline Zacatecas & 184,823 & 98.6 & 76.7 & 16.9 & 3.4 & 0.3 & 3.6 \\
\hline
\end{tabular}

Fuente: estimaciones propias a partir de la base de datos de la Encuesta Intercensal 2015. 
Tabla 4. Modos de transporte utilizados por adolescentes para ir a la escuela por entidad federativa

\begin{tabular}{|c|c|c|c|c|c|c|c|}
\hline & \multirow{2}{*}{$\begin{array}{c}\text { Total de } \\
\text { adolescentes }\end{array}$} & \multirow{2}{*}{$\begin{array}{c}\% \text { del total } \\
\text { que asisten a } \\
\text { escuela }\end{array}$} & \multicolumn{5}{|c|}{ Medio de transporte a la escuela } \\
\hline & & & $\begin{array}{c}\text { Caminando } \\
(\%)\end{array}$ & $\begin{array}{c}\text { Carro } \\
(\%)\end{array}$ & $\begin{array}{c}\text { Transporte público } \\
(\%)\end{array}$ & $\begin{array}{c}\text { Transporte escolar } \\
(\%)\end{array}$ & $\begin{array}{c}\text { Bicicleta } \\
(\%)\end{array}$ \\
\hline Aguascalientes & 111,731 & 89.1 & 58.9 & 23.3 & 16.5 & 1.4 & 2.0 \\
\hline Baja California & 247,528 & 92.9 & 42.3 & 35.8 & 22.3 & 2.1 & 0.7 \\
\hline Baja California Sur & 49,512 & 95.0 & 41.7 & 39.1 & 18.3 & 2.9 & 0.3 \\
\hline Campeche & 67,078 & 89.2 & 49.5 & 18.4 & 26.1 & 2.5 & 3.8 \\
\hline Coahuila & 228,602 & 90.6 & 40.2 & 26.3 & 27.8 & 6.5 & 0.7 \\
\hline Colima & 50,524 & 90.1 & 52.8 & 24.5 & 24.6 & 1.1 & 0.8 \\
\hline Chiapas & 454,976 & 84.2 & 74.6 & 5.2 & 18.6 & 0.6 & 1.6 \\
\hline Chihuahua & 274,553 & 88.5 & 44.2 & 32.3 & 18.0 & 6.3 & 0.4 \\
\hline Ciudad de México & 506,211 & 94.5 & 48.4 & 15.1 & 36.8 & 3.7 & 0.6 \\
\hline Durango & 138,424 & 89.4 & 60.1 & 17.1 & 22.3 & 2.1 & 0.9 \\
\hline Guanajuato & 471,930 & 88.4 & 56.9 & 15.3 & 25.1 & 1.1 & 3.6 \\
\hline Guerrero & 303,074 & 89.3 & 70.9 & 5.1 & 24.4 & 0.1 & 0.4 \\
\hline Hidalgo & 223,860 & 93.0 & 57.8 & 8.8 & 34.5 & 0.7 & 1.2 \\
\hline Jalisco & 598,800 & 88.9 & 58.2 & 18.0 & 22.7 & 2.2 & 1.6 \\
\hline México & $1,200,788$ & 92.7 & 61.1 & 8.7 & 28.0 & 3.1 & 1.2 \\
\hline Michoacán & 355,965 & 88.5 & 59.5 & 12.3 & 28.0 & 1.1 & 1.4 \\
\hline Morelos & 136,750 & 90.4 & 53.5 & 12.7 & 35.6 & 0.6 & 0.6 \\
\hline Nayarit & 86,379 & 90.9 & 66.0 & 13.0 & 21.0 & 0.4 & 1.7 \\
\hline Nuevo León & 365,998 & 93.0 & 52.4 & 26.5 & 14.8 & 7.4 & 0.3 \\
\hline Oахаса & 324,358 & 87.8 & 73.2 & 5.0 & 19.5 & 0.4 & 1.4 \\
\hline Puebla & 508,365 & 89.0 & 66.5 & 7.3 & 25.8 & 0.5 & 1.6 \\
\hline Querétaro & 156,441 & 91.4 & 49.8 & 18.9 & 27.6 & 5.5 & 0.4 \\
\hline Quintana Roo & 107,165 & 92.6 & 53.7 & 21.2 & 23.6 & 0.4 & 2.9 \\
\hline San Luis Potosí & 226,604 & 92.7 & 63.9 & 15.7 & 18.3 & 1.1 & 2.7 \\
\hline Sinaloa & 222,374 & 92.5 & 48.4 & 22.3 & 28.0 & 1.0 & 1.6 \\
\hline Sonora & 218,770 & 92.2 & 45.6 & 34.6 & 17.8 & 2.9 & 1.0 \\
\hline Tabasco & 178,788 & 92.4 & 46.5 & 13.0 & 36.4 & 0.5 & 3.3 \\
\hline Tamaulipas & 250,892 & 91.1 & 36.5 & 30.4 & 26.4 & 7.4 & 0.7 \\
\hline Tlaxcala & 100,749 & 92.0 & 59.3 & 5.9 & 35.7 & 1.1 & 1.2 \\
\hline Veracruz & 612,343 & 90.1 & 65.2 & 9.3 & 26.0 & 0.5 & 0.9 \\
\hline Yucatán & 152,600 & 92.8 & 46.4 & 20.8 & 23.6 & 1.3 & 6.3 \\
\hline Zacatecas & 123,514 & 90.6 & 69.5 & 16.6 & 10.9 & 1.3 & 3.2 \\
\hline
\end{tabular}

Fuente: estimaciones propias a partir de la base de datos de la Encuesta Intercensal 2015. 
Tabla 5. Tiempo destinado al traslado de acuerdo al modo de transporte utilizado para ir a la escuela

\begin{tabular}{|c|c|c|c|c|c|}
\hline \multirow[t]{2}{*}{ Tiempo de traslado a la escuela } & \multicolumn{5}{|c|}{ Medio de transporte a la escuela } \\
\hline & $\begin{array}{c}\text { Caminando } \\
(\%)\end{array}$ & $\begin{array}{c}\text { Carro } \\
(\%)\end{array}$ & $\begin{array}{c}\text { Transporte público } \\
(\%)\end{array}$ & $\begin{array}{c}\text { Transporte escolar } \\
(\%)\end{array}$ & $\begin{array}{c}\text { Bicicleta } \\
(\%)\end{array}$ \\
\hline $\begin{array}{l}\text { General } \\
<15 \text { minutos } \\
16-30 \text { minutos } \\
31 \text { minutos- } 1 \text { hora } \\
>1 \text { hora }\end{array}$ & $\begin{array}{c}76.8 \\
20.4 \\
2.4 \\
0.4 \\
100.0\end{array}$ & $\begin{array}{c}65.8 \\
28.2 \\
5.1 \\
0.9 \\
100.0\end{array}$ & $\begin{array}{c}29.9 \\
49.2 \\
17.4 \\
3.5 \\
100.0\end{array}$ & $\begin{array}{c}38.4 \\
43.9 \\
15.6 \\
2.1 \\
100.0\end{array}$ & $\begin{array}{c}66.5 \\
29.8 \\
3.3 \\
0.4 \\
100.0\end{array}$ \\
\hline $\begin{array}{l}\text { Escolares } \\
<15 \text { minutos } \\
16-30 \text { minutos } \\
31 \text { minutos- } 1 \text { hora } \\
>1 \text { hora }\end{array}$ & $\begin{array}{c}80.5 \\
17.5 \\
1.7 \\
0.2 \\
100.0\end{array}$ & $\begin{array}{c}68.0 \\
26.6 \\
4.7 \\
0.8 \\
100.0\end{array}$ & $\begin{array}{c}35.3 \\
47.2 \\
14.7 \\
2.8 \\
100.0\end{array}$ & $\begin{array}{c}43.2 \\
40.4 \\
14.6 \\
1.8 \\
100.0\end{array}$ & $\begin{array}{c}72.2 \\
25.5 \\
2.1 \\
0.2 \\
100.0\end{array}$ \\
\hline $\begin{array}{l}\text { Adolescentes } \\
<15 \text { minutos } \\
16-30 \text { minutos } \\
31 \text { minutos- } 1 \text { hora } \\
>1 \text { hora }\end{array}$ & $\begin{array}{c}69.6 \\
25.9 \\
3.4 \\
0.8 \\
100.0\end{array}$ & $\begin{array}{c}62.2 \\
30.9 \\
5.8 \\
1.1 \\
100.0\end{array}$ & $\begin{array}{c}26.9 \\
50.3 \\
19.0 \\
3.8 \\
100.0\end{array}$ & $\begin{array}{c}33.3 \\
47.6 \\
16.7 \\
2.5 \\
100.0\end{array}$ & $\begin{array}{c}55.6 \\
38.0 \\
5.5 \\
0.9 \\
100.0\end{array}$ \\
\hline
\end{tabular}

Fuente: estimaciones propias a partir de la base de datos de la Encuesta Intercensal 2015.

Tabla 6. Modelos de regresión lineal teniendo como variable dependiente los modos de transporte y como variable independiente las características demográficas, socioeconómicas y de seguridad pública a nivel estatal

\begin{tabular}{|c|c|c|c|c|c|c|c|c|c|c|}
\hline & \multicolumn{2}{|c|}{ Caminando } & \multicolumn{2}{|c|}{ Carro } & \multicolumn{2}{|c|}{$\begin{array}{l}\text { Transporte } \\
\text { público }\end{array}$} & \multicolumn{2}{|c|}{$\begin{array}{l}\text { Transporte } \\
\text { escolar }\end{array}$} & \multicolumn{2}{|c|}{ Bicicleta } \\
\hline & $r$ & B & $\mathbf{r}$ & B & $\mathbf{r}$ & B & $\mathbf{r}$ & B & $\mathbf{r}$ & B \\
\hline $\begin{array}{l}\text { Escolares } \\
\text { Constante } \\
\text { Inseguridad alimentaria } \\
\text { Ruralidad } \\
\text { Inseguridad pública } \\
\text { Problemas comunitarios } \\
\text { Muertes violentas }(\%)\end{array}$ & $\begin{array}{c}0.38^{*} \\
0.41^{*} \\
0.16 \\
-0.13 \\
0.12\end{array}$ & $\begin{array}{c}69.23^{* * *} \\
0.10^{*}\end{array}$ & $\begin{array}{l}-0.44^{*} \\
-0.45^{* *} \\
-0.31 \\
-0.06 \\
-0.01\end{array}$ & $\begin{array}{c}36.64^{* * *} \\
-0.12^{*} \\
-0.05^{*}\end{array}$ & $\begin{array}{c}0.25 \\
0.15 \\
0.48^{* *} \\
0.46 \\
-0.10\end{array}$ & $0.03^{* *}$ & $\begin{array}{c}-0.39^{*} \\
-0.34 \\
0.18 \\
0.30 \\
0.03\end{array}$ & $\begin{array}{l}3.88^{* * *} \\
-0.01 *\end{array}$ & $\begin{array}{c}0.90 \\
0.18 \\
-0.26 \\
0.13 \\
-0.48^{*}\end{array}$ & $-0.40^{*}$ \\
\hline $\begin{array}{l}\text { Adolescentes } \\
\text { Constante } \\
\text { Inseguridad alimentaria } \\
\text { Ruralidad } \\
\text { Inseguridad pública } \\
\text { Muertes violentas (\%) }\end{array}$ & $\begin{array}{c}0.31 \\
0.34^{\mathrm{a}} \\
0.27 \\
0.56\end{array}$ & $\begin{array}{c}55.67^{* * *} \\
0.08^{\mathrm{a}}\end{array}$ & $\begin{array}{l}-0.42^{*} \\
-0.40^{*} \\
-0.29 \\
-0.08\end{array}$ & $\begin{array}{c}31.55^{* * *} \\
-0.10^{*}\end{array}$ & $\begin{array}{c}0.25 \\
0.25 \\
0.42^{*} \\
0.13\end{array}$ & $\begin{array}{l}11.04^{\mathrm{a}} \\
0.04^{*}\end{array}$ & $\begin{array}{c}-0.44^{*} \\
-0.40^{*} \\
0.10 \\
-0.40\end{array}$ & $\begin{array}{l}5.75^{* * *} \\
-0.02^{*}\end{array}$ & $\begin{array}{c}0.08 \\
0.16 \\
-0.52 \\
-0.52^{* *}\end{array}$ & $\begin{array}{c}0.88^{*} \\
-0.03^{* *}\end{array}$ \\
\hline
\end{tabular}

r: coeficiente de correlación de Pearson; ß: coeficientes de regresión estimados a partir de modelos de regresión lineal en los que se incluyeron las variables con valores.

a $0.50<\mathrm{p}<0.100 ;{ }^{*} \mathrm{p}<0.050$; ${ }^{* *} \mathrm{p}<0.010$; ${ }^{* * *} \mathrm{p}<0.001$.

En el sexo masculino fue más frecuente el uso de bicicleta, mientras el sexo femenino solía transportarse con medios motorizados con mayor frecuencia. Estas diferencias entre sexos pueden ser atribuibles a que a los varones se les alienta a ser independientes y activos, mientras que se piensa que es más probable que las mujeres sean víctimas de violencia ${ }^{11,18,19}$. Teniendo en cuenta los altos niveles de inseguridad y feminicidios de los últimos años, es una tendencia que podría agudizarse si el problema no es tratado.

Consistente con otros estudios con niños de 5 a 7 $a_{n} 0 s^{18}$ y adolescentes de 11 a 15 años ${ }^{5}$, cuanto más 
cerca vivían los niños de la escuela era más frecuente que se utilizaran medios de transporte activos. Esta relación fue evaluada con el tiempo de traslado. Sin embargo, más adelante convendría hacer la relación con la distancia en kilómetros.

Las características socioeconómicas de la familia intervienen en la elección de los modos de transporte, ya que el uso de medios activos fue mayor cuando la escolaridad del jefe de familia era menor; en contraparte, con estudios de nivel superior, usaban más transporte pasivo. Una de las principales razones de esta diferencia es la mayor probabilidad de que los niños en condiciones de pobreza caminen pese a las condiciones adversas del entorno, ya que el uso de transporte activo no implica un gasto adicional, mientras que las familias con mejor posición socioeconómica pueden adquirir automóviles o pagar el transporte motorizado. Aunque también es posible que el uso de automóvil ahora sea un medio de obtener estatus social, lo que lleva a su uso injustificado - desde el punto de vista de la salud-, sobre todo cuando se trata de distancias cortas. Si el último factor tiene peso en la decisión de utilizar medios motorizados, convendría diseñar intervenciones para desalentar su uso.

En los estados donde más población residía en áreas rurales y aquellos de la región sur (Guerrero, Oaxaca y Chiapas) fue más frecuente que escolares y adolescentes utilizaran modos de transporte activo (i.e., caminar o usar bicicleta). Por el contrario, en las entidades más urbanizadas (por ejemplo, Ciudad de México) el uso de modos de trasporte pasivo es mayor. En congruencia con lo anterior, en los modelos de regresión lineal, la ruralidad se relacionó positivamente con la frecuencia de caminar en ambos grupos de edad, pero negativamente con el uso de automóvil en escolares. Una explicación a lo anterior es que en las ciudades existen más incentivos para el uso de transportes que reduzcan el tiempo de traslado, lo cual favorece el uso de transporte motorizado. Por ejemplo, en la Ciudad de México, el uso de transporte pasivo fue de los más altos en escolares y adolescentes. Estos resultados sugieren que los usos de estos modos de transporte están condicionados por las condiciones socioeconómicas de las localidades y la disponibilidad de transporte motorizado. También indican la necesidad de concentrar los esfuerzos para desalentar el uso de transporte motorizado en las ciudades.

El uso de bicicleta en los dos grupos de edad, además, estuvo relacionado negativamente con muertes violentas. De este modo, los estados donde existe menos frecuencia de uso de bicicleta (por ejemplo, Ciudad de México, Guerrero, Nuevo León) son en los que existen altas tasas de muertes violentas. Además, en las entidades más inseguras (Tamaulipas, Guerrero, Estado de México) fue más frecuente el uso de auto, transporte público y escolar. Ello explica por qué la inseguridad pública tiene relación positiva con el uso de transporte público, pero negativa con el uso de automóvil. Estos hallazgos podrían indicar que la inseguridad pública puede estar incidiendo en la forma de transportarse de niños y adolescentes a la escuela. Al respecto, en Australia ${ }^{11}$, California ${ }^{18}$, Inglaterra y Alemania $^{19}$, el temor de los padres por la inseguridad pública se asocia con el menor uso de modos de transporte activo. En México, de acuerdo con la última emisión de la ENVIPE ${ }^{13}$, el $75.7 \%$ de las personas mayores de 18 años tienen miedo a ser víctimas de al menos un delito; cabe destacar que los datos presentados en dicha encuesta incluyen información de la denominada «cifra negra", que son los delitos que suelen no denunciarse por desconfianza en los servicios de seguridad. Lo anterior supone que, a pesar de que el medio social resulte inseguro o peligroso, las personas aún prefieren el uso de transporte masivo como modo de traslado.

Los resultados de nuestro estudio deben interpretarse a la luz de sus limitaciones. Si bien el análisis ecológico reportado permite generar hipótesis, también debe reconocerse que está sujeto a los siguientes sesgos: no es posible extender los hallazgos a nivel individual, no se pueden controlar variables de confusión y es difícil determinar la secuencia temporal entre las variables independientes y dependientes.

En síntesis, los resultados obtenidos indican que el contexto social define el tipo de transporte que utilizan escolares y adolescentes: por un lado, la urbanización y la mayor riqueza de los estados se relacionan con el uso de modos de transporte pasivos; por otro lado, la inseguridad pública también propicia el abandono del transporte activo y su sustitución por medios de transporte pasivo. Dado su papel en la transición epidemiológica, la inactividad física es un factor que es relevante, por lo que es necesario que se favorezca un entorno propicio para su reducción. Deben desarrollarse políticas públicas, no solo para promover que la población sea más activa, sino, además, para asegurar que en el proceso no se ponga en riesgo su integridad mental y física. En este sentido, las estimaciones que se presentaron a nivel estatal pueden ser de utilidad a las autoridades federales y locales para identificar áreas de oportunidad específicas de su contexto. 


\section{Responsabilidades éticas}

Protección de personas y animales. Los autores declaran que para esta investigación no se han realizado experimentos en seres humanos ni en animales.

Confidencialidad de los datos. Los autores declaran que han seguido los protocolos de su centro de trabajo sobre la publicación de datos de pacientes.

Derecho a la privacidad y consentimiento informado. Los autores declaran que en este artículo no aparecen datos de pacientes.

\section{Conflicto de intereses}

Los autores declaramos que no existe ningún conflicto de interés.

\section{Financiamiento}

No se contó con financiación externa para la realización del presente manuscrito.

\section{Bibliografía}

1. Hume C, Timperio A, Salmon J, Carver A, Giles-Corti B, Crawford D Walking and cycling to school: predictors of increases among children and adolescents. Am J Prev Med. 2009;36:195-200.

2. Oviedo G, Sánchez J, Castro R, Calvo M, Sevilla JC, Iglesias A, et al. Niveles de actividad física en población adolescente: estudio de caso. Retos. 2015;23:43-7.

3. United States. Department of Health and Human Services. Physical activity and health: a report of the Surgeon General. Atlanta; 1996. Disponible en: https://www.cdc.gov/nccdphp/sgr/index.htm.

4. Larouche R, Saunders TJ, Faulkner G, Colley R, Tremblay M. Associations between active school transport and physical activity, body composition, and cardiovascular fitness: a systematic review of 68 studies. $J$ Phys Act Health. 2014;11:206-27.
5. Buliung RN, Mitra R, Faulkner G. Active school transportation in the Greater Toronto Area, Canada: an exploration of trends in space and time (1986-2006). Prev Med. 2009;48:507-12.

6. Baig F, Hameed MA, Li M, Shorthouse G, Roalfe AK, Daley A. Association between active commuting to school, weight and physical activity status in ethnically diverse adolescents predominately living in deprived communities. Pub Health. 2009;123:39-41.

7. Davison KK, Werder JL, Lawson CT. Children's active commuting to school: current knowledge and future directions. Prev Chronic Dis. 2008;5:A100.

8. Timperio A, Ball K, Salmon J, Roberts R, Giles-Corti B, Simmons D, et al Personal, family, social, and environmental correlates of active commuting to school. Am J Prev Med. 2006;30:45-51.

9. Shamah-Levy T, Cuevas-Nasu L, Rivera-Dommarco J, Hernández-Ávila M. Encuesta Nacional de Salud y Nutrición de Medio Camino 2016. Informe final de resultados. Secretaría de Salud, Instituto Nacional de Salud Pública; 2016. Disponible en: https://www.gob.mx/cms/uploads/attachment/file/209093/ENSANUT.pdf.

10. Jauregui A, Medina C, Salvo D, Barquera S, Rivera-Dommarco JA. ACtive commuting to school in Mexican adolescents: evidence from the Mexican National Nutrition and Health Survey. J Phys Act Health. 2015; 12:1088-95.

11. Foster S, Villanueva K, Wood L, Christian H, Giles-Corti B. The impact of parents' fear of strangers and perceptions of informal social control on children's independent mobility. Health Place. 2014;26:60-8.

12. Encuesta Intercensal 2015. Disponible en: http://www.inegi.org.mx/est/ contenidos/Proyectos/encuestas/hogares/especiales/ei2015/

13. Encuesta Nacional de Victimización y Percepción sobre Seguridad Pública 2016. Disponible en: https://www.inegi.org.mx/programas/envipe/2016/.

14. Instituto Nacional de Estadística y Geografía. Mortalidad (Causas de defunción. Muertes accidentales y violentas hasta 2014). Disponible en: https://www.inegi.org.mx/sistemas/olap/Proyectos/bd/continuas/mortalidad/MortalidadGeneral.asp

15. Indicadores de víctimas visibles e invisibles de homicidio. [en proceso de publicación]. 2012.

16. Secretaría de Salud. Panorama epidemiológico y estadístico de la mortalidad en México 2011. México: Dirección de Información Epidemiológica; 2015. Disponible en: https://www.gob.mx/salud/documentos/panorama-epidemiologico-y-estadistico-de-la-mortalidad-en-mexico-2011.

17. Secretariado Ejecutivo del Sistema Nacional de Seguridad Pública. Tasas por cada 100 mil habitantes 1997-2016. Disponible en: http://secretariadoejecutivo.gob.mx/docs/pdfs/tasas $\% 20$ por\%20cada\%20100\%20 mil\%20habitantes/Tasas072017.pdf.

18. Su JG, Jerrett M, McConnell $R$, Berhane $K$, Dunton $G$, Shankardass $K$, et al. Factors influencing whether children walk to school. Health Place. 2013;22:153-61

19. Shaw B, Watson B, Frauendienst B, Redecker A, Jones T, Hillman M. Children's Independent Mobility: A Comparative Study in England and Germany (1971-2010). Londres: Policy Studies Institute; 2013. Disponible en: http://www.psi.org.uk/site/publication detail/852. 